\title{
Umbilical Endometriosis: Case Report and Review of Current Management
}

\author{
Akhter F, Mallick R*, Ball E \\ Royal London Hospital, London, England, UK \\ *Corresponding Author: Mallick R, Royal London Hospital, London, England, UK, Tel: 07411617143; E-mail: \\ rmallick@doctors.org.uk
}

Received: 02 May 2018; Accepted: 16 May 2018; Published: 18 May 2018

\begin{abstract}
Umbilical endometriosis (UE) remains a rare occurrence with an incidence of approximately $0.5-1.0 \%$ in all cases of endometriosis. It can be subdivided into primary UE (Villar's nodule), developing spontaneously, or secondary following previous abdominal surgery and is usually due to the iatrogenic seeding of endometrial tissue. We present the case of a 35-year-old patient who presented with a large umbilical nodule that was successfully treated with complete surgical excision and reconstruction of the umbilicus and review the wider literature.
\end{abstract}

Keywords: Umbilical; Endometriosis

\section{Introduction}

Endometriosis is defined as the presence of endometrial tissue out with the uterine cavity and it affects between 6$10 \%$ of all women of reproductive age [1]. The areas most commonly affected include the uterosacral ligaments, recto-sigmoid colon, recto-vaginal septum, vagina and bladder. Endometriosis can also develop in areas out with the pelvis of which the umbilicus is the comment cutaneous site, however, it still remains a rare occurrence with an incidence of approximately $0.5-1.2 \%$ in all cases of endometriosis [2]. Primary umbilical endometriosis was first described by Villar in 1886, and it represents $75 \%$ of all cases of umbilical endometriosis [2]. We describe a case of secondary umbilical endometriosis, developing after a previous laparoscopy, and our surgical approach to management involving radical excision with a laparoscopic assisted approach.

\section{Case Report}

A 35-year-old patient presented to the gynaecology clinic with a history of worsening pelvic pain and cyclical umbilical bleeding on a background of grade 4 endometriosis, having undergoing a laparoscopy and excision of the endometriosis 2 years previously. Examination revealed a $3 \mathrm{~cm}$ tender nodule in the umbilicus, a fixed uterus and 
retro-cervical tenderness. Subsequent MRI suggested an umbilical endometriotic nodule with retro-cervical endometriosis and findings at the time of her laparoscopy were of grade 4 endometriosis and a $3 \mathrm{~cm}$ umbilical endometriotic nodue (Figure 1). Following laparoscopic excision of her pelvic endometriosis the umbilical nodule was excised. A circumferential incision was made around the nodule using diathermy, ensuring full excision with good margins. The underlying fascia was closed using 2/0 polyglactin (Vicryl ${ }^{\circledR}$ ) sutures and the umbilicus was then reconstructed using 2/0 poliglecaprone (Monocryl ${ }^{\circledR}$ ) sutures giving a good aesthetic result. The whole procedure was performed with laparoscopic assistance to ensure adequate excision and complete closure of the excised area. Histology confirmed endometriosis and follow up 6 months later confirmed a good aesthetic result with no evidence of recurrence.

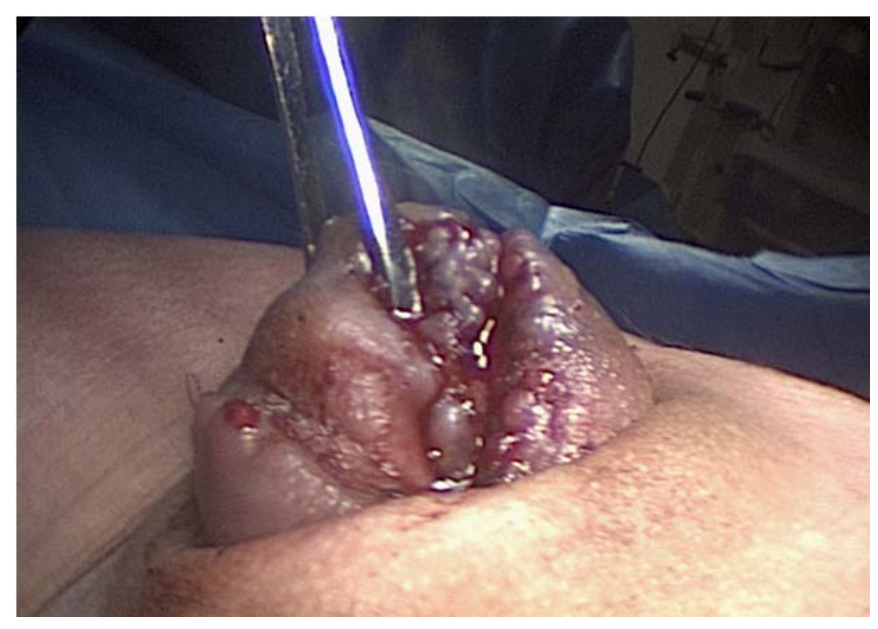

Figure 1: Endometriotic umbilical nodue.

\section{Discussion}

Primary umbilical endometriosis, the more common form of umbilical endometriosis, develops spontaneously in the absence of any previous abdominal surgery. The exact pathogenesis is unknown, however possible theories include the spread of endometrial cells to the umbilicus through the abdominal cavity, via the lymphatic system or through the embryonic remnants in the umbilical folds [3-5]. Secondary umbilical endometriosis develops following previous surgery (caesarean section, abdominal hysterectomy, appendicectomy, laparoscopy etc.) Scholefield et al. [6] and is due to iatrogenic seeding of endometrial tissue. The clinical features include an umbilical swelling (90\%), often associated with cyclical pain (81.5\%) and bleeding (49.2\%). The differential diagnoses include granuloma, umbilical polyps, haemangioma, melanocytic nevus, seborrhoeic keratosis, granular cell tumour and umbilical hernia. Investigations may include an ultrasound to assess the echogenicity and vascular involvement. MRI can also be helpful, although no imaging modality is truly diagnostic. Medical treatments such as the contraceptive pill or GnRH analogues are an option and can be effective in diminishing symptoms temporarily, however after cessation of hormonal treatment symptom recurrence is common [1]. The definitive management involves surgery, which can vary from superficial diathermy to the more radical omphalectomy with a concomitant laparoscopic approach. This 
allows for total excision of the umbilicus and repair of the underlying fascia and reconstruction of the umbilicus. Another option is local resection of the endometriotic tissue and sparing of the umbilicus. Whilst there is no real data on follow up of these surgically treated cases, Fedele et al. [7] described in their case series recurrence of umbilical endometriosis in those managed with superficial surgical approach [7]. They described a radical en bloc excision of the umbilicus including the fascia and perotineum, with subsequent histology confirming intrafascial endometriosis in all of those cases. This would support a more radical approach to surgery aided by laparoscopy to enable complete excision of umbilical endometriosis and reduce local recurrence. The use of laparoscopy also allows the extent of the nodule to be explored and ensures adequate excision of the lesion and the accurate reconstruction of the fascial planes, whilst also allowing concomitant pelvic disease to be treated.

\section{Declaration of Interest}

The authors report no declaration of interest

\section{References}

1. Giudice LC, Kao LC. Endometriosis. Lancet 364 (2004): 1789-1799.

2. Victory R, Diamond MP, Johns DA. Villar's nodule: a case report and systematic literature review of endometriosis externa of the umbilicus. J Minim Invasive Gynecol 14 (2007): 23-32.

3. Efremidou EI, Kouklakis G, Mitrakas A, et al. Primary umbilical endometrioma: a rare case of spontaneous abdominal wall endometriosis. Int J Gen Med 5 (2012): 999-1002.

4. Theunissen CIJM, Ijpma FFA. Primary umbilical endometriosis: a cause of a painful umbilical nodule. Journal of Surgical Case Reports (2015): rjv025.

5. Fancellu A, Pinna A, Manca A, et al. Primary umbilical endometriosis. Case report and discussion on management options. Int J Surg Case Rep 4 (2013): 1145-1148.

6. Scholefield HJ, Sajjad Y, Morgan PR. Cutaneous endometriosis and its association with caesarean section and gynaecological procedures. J Obstet Gynaecol 22 (2002): 553-554.

7. Fedele L, Frontino G, Bianchi S, et al. Umbilical endometriosis: a radical excision with laparoscopic assistance. Int J Surg 8 (2010): 109-111.

Citation: Akhter F, Mallick R, Ball E. Umbilical Endometriosis: Case Report and Review of Current Management. Obstetrics and Gynecology Research 1 (2018): 072-074.

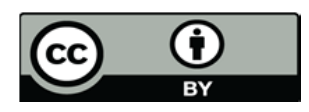

This article is an open access article distributed under the terms and conditions of the Creative Commons Attribution (CC-BY) license 4.0 